\title{
Microlens systems for fluorescence detection in chemical microsystems
}

\author{
Jean-Christophe Roulet \\ Reinhard Völkel \\ Hans Peter Herzig \\ Elisabeth Verpoorte \\ Nico F. de Rooij \\ René Dändliker, MEMBER SPIE \\ University of Neuchâtel \\ Institute of Microtechnology \\ rue A.-L Breguet 2 \\ $\mathrm{CH}-2000$ Neuchâtel, Switzerland \\ E-mail: jean-christophe.roulet@imt.unine.ch
}

\begin{abstract}
Micro-optical systems based on refractive microlenses are investigated. These systems are integrated on a chemical chip. They focus an excitation beam into the detection volume (microliter or even submicroliter scale) and collect the emitted light from fluorescent molecules. The fluorescence must be carefully separated by spatial and spectral filtering from the excitation. This paper presents the ray tracing simulation, fabrication, and measurement of three illumination systems. The measurements show that an adroit placement and combination of microfabricated lenses and stops can increase the separation between the excitation light and the fluorescence light. Moreover we present the successful detection of a $20 \mathrm{nM}$ Cy5 ${ }^{\mathrm{TM}}$ (Amersham Life Science Ltd.) solution in a 100- $\mu \mathrm{m}$-wide and 50- $\mu \mathrm{m}$-deep microchannel (excitation volume $\approx 250 \mathrm{pL}$ ) using one of these illumination systems. The microchemical chip with the micro-optical system has a thickness of less than $2 \mathrm{~mm}$. (c) 2001 Society of Photo-Optical Instrumentation Engineers. [DOI: 10.1117/1.1359522]
\end{abstract}

Subject terms: biomedical optics; fluorescence; micro-optics; microlenses.

Paper 200007 received Jan. 5, 2000; revised manuscript received Nov. 16, 2000; accepted for publication Dec. 5, 2000.

\section{Introduction}

Microfabrication technology has become a widely used approach in the development of miniaturized systems for chemical analysis in microfluidic channels. ${ }^{1-6}$ In such systems one of the most commonly used modes of detection is fluorescence, due to its selectivity and high sensitivity. ${ }^{7,8}$ The successful implementation of the detection system relies on three factors. First, an excitation beam must be focused into the tiny detection volume (less than $10^{-3} \mathrm{~mm}^{3}$, or $1 \mathrm{~nL}$ ). The fluorescence light must then be collected and separated from the unabsorbed excitation light at the incident wavelength. Finally, the fluorescence light must be transported to a detector. This is challenging for the detection system, because the optical components must be precisely aligned with respect to a microchannel or microwell which has a width on the order of tens of micrometers. The problem becomes even more complicated when detection in an array of closely spaced microfluidic channels is desired. Presently, the most commonly used strategy is to scan the analytical chip with respect to a stationary optical detection system (such as a confocal microscope) or by sweeping the channels with a light beam. ${ }^{9}$ These solutions require precise and thus often expensive and bulky mechanical systems, such as translation stages or movable mirror systems, which are almost impossible to integrate and therefore not suitable for the realization of a micrototal-analysis system ( $\mu$ TAS).

The aim of this paper is to study micro-optical systems for fluorescence detection that can eventually be integrated directly onto chips. For that purpose, we simulated and built three illumination systems composed of refractive microlenses. We show how adroit placement and utilization of microlenses make it possible to focus an excitation beam into a tiny volume while allowing for fluorescence light collection and its separation from the transmitted (unabsorbed) excitation light. Moreover, the micro-optical fabrication method is compatible with the microfabrication techniques (photolithography, chemical wet etching) commonly used to realize microfluidic networks on chip (chemical chip). ${ }^{10-13}$ Integrated detection systems for $\mu$ TAS can therefore be realized by these means.

\section{Detection Principle}

Although some molecules are naturally fluorescent (e.g., riboflavin, vitamin B2), the fluorescence emission is often rather weak, and most molecules in fact do not fluorescence at all. For this reason, fluorescent labels (or fluorochromes) are often chemically attached to the substance to be detected. When the analyte containing the fluorescent molecules is illuminated at an appropriate wavelength, fluorescence light is generated at a lower energy (longer wavelength). Absorption and emission spectra are specific to each fluorescent molecule. The difference between the excitation peak and the fluorescence peak ranges from 7 to $238 \mathrm{~nm} .{ }^{14}$ The extinction coefficient $\epsilon$, the quantum yield $\phi$, and the fluorescence lifetime $\tau$ are the three main fluorochrome parameters. The extinction coefficient represents the probability of absorption of excitation photons by the fluorescent molecules. Useful fluorochromes have an excitation coefficient ranging from 40,000 to 250,000 $\mathrm{M}^{-1} \mathrm{~cm}^{-1}$. The quantum yield is defined as the number of quanta emitted divided by the number of quanta absorbed. For practical purposes, the quantum yields should be greater than 0.1 . The fluorescence lifetime is the average time that a molecule remains in the excited state. It ranges from $100 \mathrm{~ns}$ down to $1 \mathrm{~ns} .{ }^{15}$ 


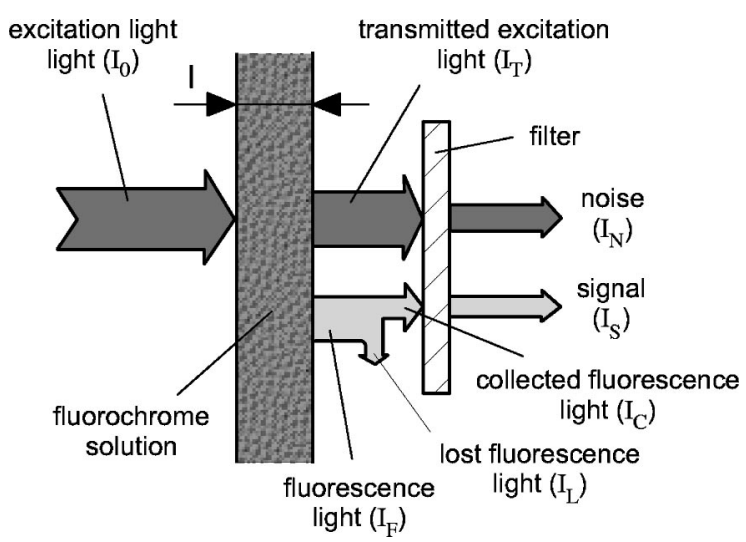

Fig. 1 Separation scheme. Part of the excitation light $I_{0}$ is absorbed by the fluorochrome in solution and used to generate fluorescence light $I_{F}$. Here $I_{C}$ represents the part of the fluorescence light that is collected, and $I_{L}$ the part that is lost. After the interference filter, the fluorescence light intensity $I_{S}$ must be greater than the remaining excitation light $I_{N}$.

The absorbance of a solution containing fluorescent molecules is defined by the Lambert-Beer law, ${ }^{16,17}$

$A=\log \frac{I_{0}}{I_{T}}=\epsilon l c$,

where $I_{0}$ is the incident excitation intensity, $I_{T}$ is the transmitted excitation intensity, $c$ is the concentration of the fluorescent molecules per unit volume (usually given in moles per liter), and $l$ is the path length of the excitation light through the sample. The Lambert-Beer law describes correctly the absorbance for fluorochrome concentrations of $0.01 \mathrm{M}$ or lower. Above this concentration, interaction between absorbing molecules reduces the absorbance. ${ }^{17}$

The amount of excitation light absorbed by fluorescent molecules can be calculated with Eq. (1). Since the fluorescence light intensity $I_{F}$ is proportional to the amount of absorbed excitation light $\left(I_{0}-I_{T}\right)$ and to the quantum yield, ${ }^{18}$ it can be expressed by

$I_{F}=\phi I_{0}\left(1-10^{-A}\right)$.

By combining Eqs. (1) and (2), the ratio of the fluorescence to the transmitted light intensity becomes

$\frac{I_{F}}{I_{T}}=\phi\left(10^{A}-1\right)$

In the case of a $10-\mathrm{nM}$ solution of $\mathrm{Cy}^{\mathrm{TM}}$ (cyanine dye molecules, $\phi>0.28, \epsilon=250,000 \mathrm{M}^{-1} \mathrm{~cm}^{-1}$ ) in a detection cell $25 \mu \mathrm{m}$ deep, the ratio $I_{F} / I_{T}$ is about $4 \times 10^{-6}$. The fluorescence intensity is thus about 250,000 times weaker than the excitation intensity.

Therefore, fluorescence detection cannot be performed without separating the fluorescence light from the transmitted excitation light. Interference filters are commonly used for that purpose (see Fig. 1). In the ideal case where all the fluorescence light was collected (a situation which is hardly conceivable because of the uniform angular emission of the fluorescence light), the lost fluorescence light $I_{L}$ would be
0 . Thus, the ratio between the fluorescence light $I_{S}$ and the transmitted excitation light $I_{N}$ remaining after the interference filter would be given by

$\frac{I_{S}}{I_{N}}=\phi\left(10^{A}-1\right) 10^{\mathrm{OD}(e)-\mathrm{OD}(f)}$,

where $\mathrm{OD}(f)$ is the optical density of the filters at the fluorescence wavelength (mean value) and $\operatorname{OD}(e)$ is the optical density at the excitation wavelength. A commercial filter specifically designed for $\mathrm{Cy} 5^{\mathrm{TM}}$ detection has a value of $27 \times 10^{-3}$ (maximal transmission of $94 \%$ ) for $\mathrm{OD}(f)$ and a value of about 5 (transmission less than $10^{-6} \%$ ) for $\mathrm{OD}(e)$. Although this is an ideal case, the fluorescence light intensity is still about 2.6 times weaker than the transmitted excitation intensity.

In most cases, the fluorescence light is collected by means of an objective with a limited numerical aperture $\left(I_{L} \neq 0\right)$. The ratio between the total amount of fluorescence light emitted from the detection volume $\left(I_{F}\right)$ and the collected fluorescence light $I_{C}$ is then given by

$\frac{I_{C}}{I_{F}}=\frac{I-\cos \theta}{2}$,

where $\theta$ is the acceptance angle of the detection system. On replacing $\theta$ with the numerical aperture NA, Eq. (5) becomes

$\frac{I_{C}}{I_{F}}=\frac{1-\left(1-\mathrm{NA}^{2} / n^{2}\right)^{1 / 2}}{2}$,

where $n$ is the refractive index of the medium before the collection lens, or objective. On inserting (6) into (4), the ratio between $I_{S}$ and $I_{N}$ now becomes

$\frac{I_{S}}{I_{N}}=\phi\left(10^{A}-1\right) 10^{\mathrm{OD}(e)-\mathrm{OD}(f)} \frac{1-\left(1-\mathrm{NA}^{2} / n^{2}\right)^{1 / 2}}{2}$.

Even for an objective with the unusually high numerical aperture of 0.9 (typical NAs for confocal systems are 0.3 to 0.8 ), the ratio of $I_{S}$ to $I_{N}$ is reduced to about 0.1 . This means that the fluorescence intensity collected with the objective is about 10 times smaller than the transmitted excitation intensity. This is reduced even further, to a value of $4 \times 10^{-3}$, for a NA of 0.3 , which is a typical value for a refractive microlens.

Of course, a part of the transmitted excitation that is scattered at system interfaces (e.g. at the microchannel walls) is not collected by the objective either. This will slightly increase the ratio of $I_{S}$ to $I_{N}$. However, spectral separation alone remains insufficient, so that the separation between the fluorescence and the unabsorbed excitation light must be improved. Angular separation or the fluorochrome emission lifetime may be exploited for this purpose. The short fluorescence lifetime of most fluorochromes makes it difficult to apply time-resolved detection, and requires sophisticated acquisition systems with very fast photodetectors. ${ }^{19,20}$ For this reason we chose the com- 


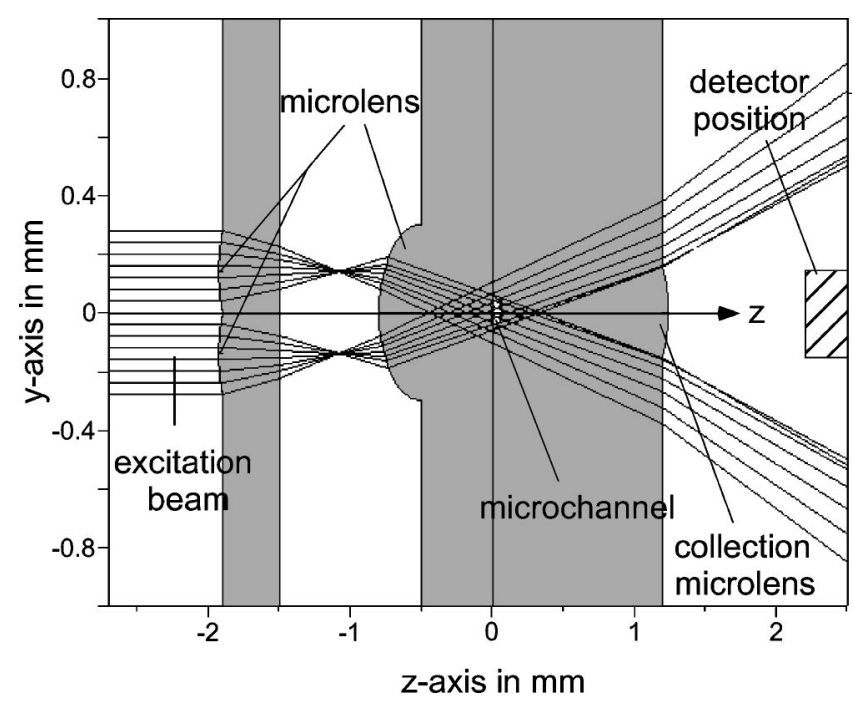

Fig. 2 2-D ray-trace simulation of beam-splitting illumination with two layers of microlenses. The first two microlenses are $280 \mu \mathrm{m}$ wide (focal length $649 \mu \mathrm{m}$ ), and the third microlens, on the second layer, is $600 \mu \mathrm{m}$ wide (focal length $800 \mu \mathrm{m}$ ).

bination of spectral and angular separation as our approach for fluorescence detection in microfluidic systems.

\section{Separation of Fluorescence Light and Transmitted Excitation Light}

The separation of fluorescence light and transmitted excitation light is a relatively easy task with standard optical components such as microscope objectives, beamsplitters, or dichroic mirrors and interference filters. They can be easily combined to realize illumination and collection systems that are perpendicular to one another, or on the same side of the chip as in confocal microscopy ${ }^{21}$ In these cases, $I_{T}$ entirely composed of back- and side-scattered excitation light, which can be easily eliminated by the interference filter. Unfortunately, beamsplitters and dichroic mirrors cannot be integrated into microfluidic devices. Thus, the excitation and collection systems must be placed on the same side of the microchemical chip. Furthermore, the surface occupied by the excitation system is lost for the collection system, which reduces either the amount of fluorescence light collected, and hence the system sensitivity, or the number of channels per chip.

The other solution consists in realizing a stacked system with the excitation system on one side of the chemical chip and the detection system on the other side. In this case, the false light is mainly transmitted excitation light. Its intensity is more important, thus requiring a better separation. Thus we combine stacks of microlenses and stops to realize excitation configurations that create an angular separation between the unabsorbed excitation light and the fluorescence light. As shown in the ray-trace simulations in Figs. $2-4$, these excitation systems make it possible not only to focus the excitation beam into the detection cells, but also to reduce the remaining excitation intensity at the detector position by blocking or directing sideways the transmitted excitation beam. Optimization of these systems would require a maximum excitation intensity in the microchannel, a maximum separation between fluorescence and excitation

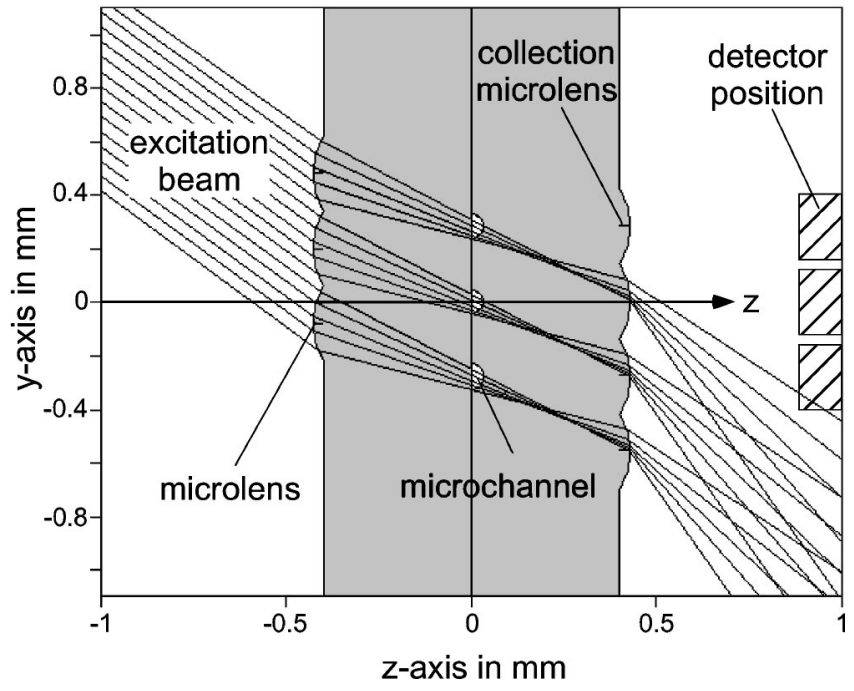

Fig. 3 2-D ray-trace simulation of an off-axis illumination. The microlenses on both layers are $280 \mu \mathrm{m}$ wide (focal length $700 \mu \mathrm{m}$ ).

light, and a numerical aperture of the collection microlens as large as possible. The microlens focal length can be fixed with a precision of $\pm 2 \%$ during the microfabrication process. ${ }^{22}$ Ideally, the microlens diameter and focal length would be adjusted to the thickness of the glass substrates in which the microchannels are formed, to ensure a maximum excitation intensity in the channel. Moreover, the size of these optical elements (of the order of a few hundred micrometers) allows the fabrication of a large number of detection systems, which is one of the major milestones for $\mu$ TAS technology.

Figure 2 illustrates a beam-splitting configuration. As this cross-sectional view shows, the illumination system is composed of two layers of cylindrical microlenses. The first two lenses split the incoming beam of light, while the third one focuses the two resulting beams into the detection cell or microchannel. The beams diverge as they leave the

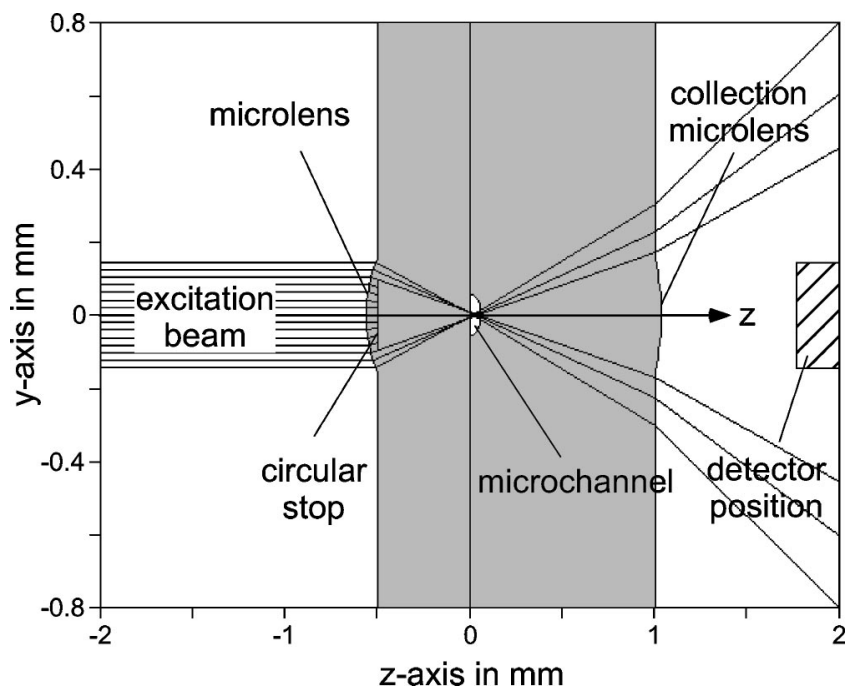

Fig. 4 2-D ray-trace simulation of an central-stop illumination system. The microlenses is $310 \mu \mathrm{m}$ wide (focal length $550 \mu \mathrm{m}$ ). The stop has a diameter of $180 \mu \mathrm{m}$. 


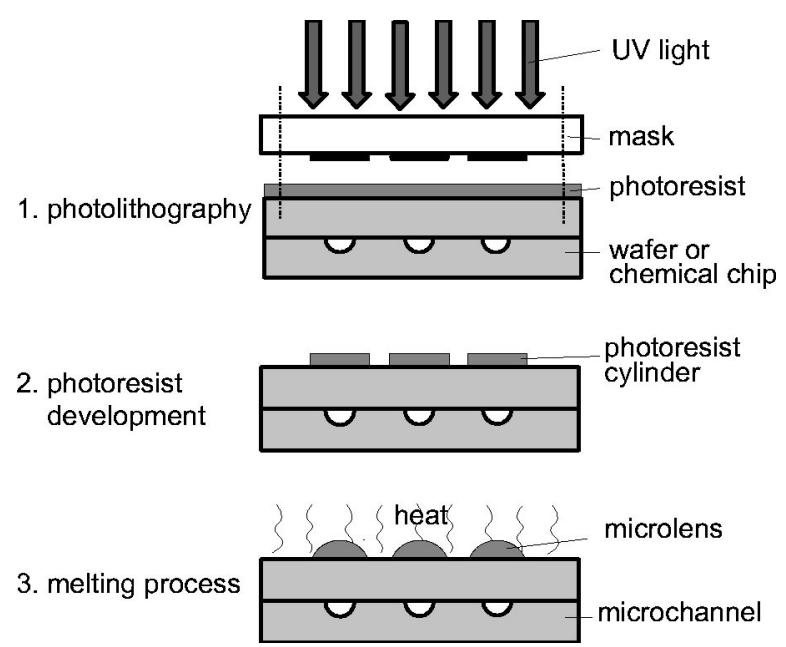

Fig. 5 Main fabrication steps for deposition of a microlens array on a glass or quartz chemical chip.

chip, producing a dark area a few millimeters behind the chip where a collection microlens or a photodetector could be placed. The off-axis illumination scheme is another possibility. In this case, the incoming beam forms an angle of $45 \mathrm{deg}$ with respect to the surface of the microchip. As can be seen from the ray tracing diagram in Fig. 3, a first layer of microlenses splits and focuses the beam into several detection microchannels. A second layer of microlenses, deposited on the other side of the microchip, directs the light sideways and also collects and focuses the fluorescence light onto the detector. The ray tracing diagram of Fig. 4 presents a third layout. A circular stop blocks the central part of the excitation beam. As in the first case, the light is focused into the microchannel and diverges as it leaves the microchip. A collection microlens and/or a detector would be placed in the dark area. The optical effect of the microchannels in Figs. 2-4 has been neglected, because only the illumination system has been realized for the experimental comparison. Ray tracing was not carried out for the collection of the fluorescence light.

These three illumination systems can be based on either cylindrical or spherical microlenses. The fabrication method, as described in the next paragraph, remains the same. The main difference is the shape of the light spot inside the microchannel. For spherical microlenses the spot is circular, whereas for cylindrical microlenses it is a line. A focal line, which permits the excitation of a complete microchannel section, could be a way to increase the illumination volume for detection of low fluorochrome concentrations. $^{23}$

\section{Fabrication Method}

Figure 5 shows the fabrication method for refractive microlenses. It is based on photolithographic technology, but this kind of microlenses can also be obtained with other techniques such as direct writing or hot embossing. ${ }^{24}$ A layer of photoresist is first deposited either on a glass or a quartz chemical chip. The microlenses can also be deposited on independent wafers, which will be placed and aligned (e.g., with a flip chip bonder) on the chemical chip afterwards. The photosensitive layer is then exposed through a chro-

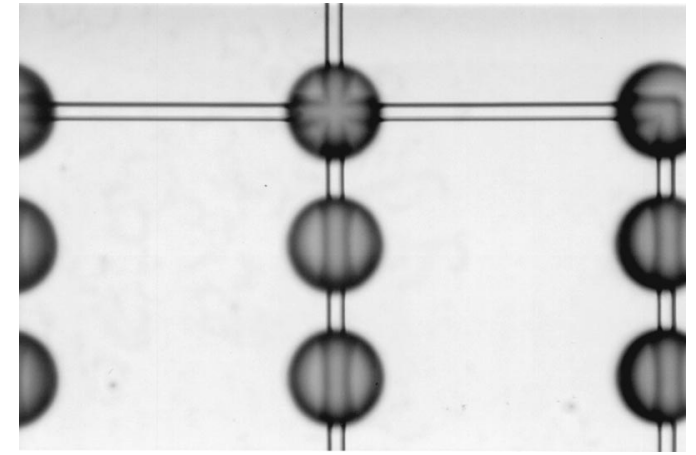

Fig. 6 Top view of photoresist spherical microlenses (slightly defocused) deposited on a sealed chemical chip. A magnified image of a microchannel can be seen in every microlens. The microlenses are $310 \mu \mathrm{m}$ wide and about $33 \mu \mathrm{m}$ high. The microchannels are $50 \mu \mathrm{m}$ deep and $110 \mu \mathrm{m}$ wide.

mium mask with the help of a mask aligner. Once developed, the chip is heated up in an oven at about $150^{\circ} \mathrm{C}$. There, the photoresist melts, and under the influence of surface tension, the microlenses are formed. Depending on the base shape of the photoresist cylinder, various kinds of microlenses can be obtained. ${ }^{22}$ Spherical microlens arrays of diameter as small as $2 \mu \mathrm{m}$ and as large as several millimeters can be produced by means of this technique. Figure 6 shows $310-\mu \mathrm{m}$-wide photoresist microlenses on a glass chip.

It is also possible to add apertures and stops directly on the same wafer, as shown on the left photograph of Fig. 11 (Sec. 5). In that case, the fabrication process begins with a low-pressure chemical vapor deposition (LPCVD) of a metallic or absorbing layer on the surface of the chemical chip or the wafer. This layer is then patterned by photolithographic and wet etching techniques. Finally, the microlenses are fabricated as described above. Alignment marks made on the wafer during the photolithographic steps for fabrication of the microlenses and apertures allow precise positioning. ${ }^{13,25}$

\section{Results}

All three illumination concepts presented in Figs. 2-4 have been realized with off-the-shell components (i.e., microlenses optimized for other applications). The microlens specifications and, in the case of the beam-splitting system, the gap between the first and the second microlens wafer have been chosen to maximize the excitation intensity in the microchannel. The ray tracing simulations were performed with the assumption that the difference between the refractive indices of the melted photoresist and the glass $(\approx 1.62$ and $\approx 1.54$, respectively, at $633 \mathrm{~nm})$ is negligible. A $10-\mathrm{mW}$ He-Ne laser was used for the excitation. The beam was expanded and collimated. The intensity distribution behind the systems was measured by means of a diffusing screen placed in front of a CCD camera. The beam-splitting scheme is composed of cylindrical microlenses on two 25$\mathrm{mm} \times 25-\mathrm{mm}$ glass plates of $0.5-\mathrm{mm}$ thickness. The plates were assemble by precise alignment under a microscope, followed by bonding using a UV-curable glue. Glass spacers and a transparent sheet, on which a pattern of apertures was printed, were inserted between the two plates. The pur- 


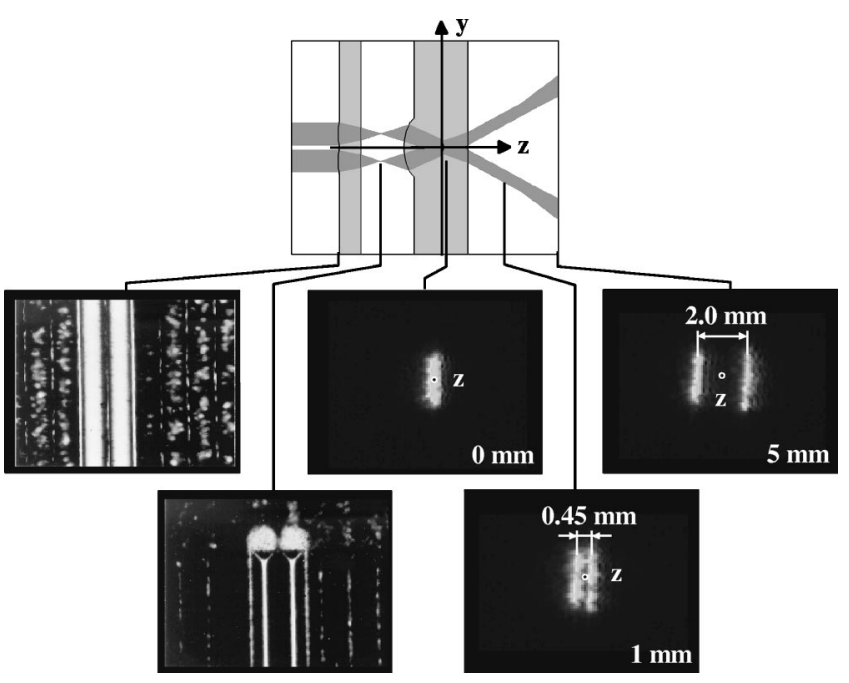

Fig. 7 Top picture: illumination scheme of the beam-splitting illumination system. The five bottom pictures show (from left to right): first layer of cylindrical microlens, focal lines situated between the two optical layers, focus spot (detection cell), and diverging beams at 1 and $5 \mathrm{~mm}$ away from the detection cell.

pose of this pattern was to block the light passing around the microlenses. The photographs in Fig. 7 show the first plate with the two cylindrical microlenses and the intensity distribution of the excitation beam in a plane perpendicular to the $z$ axis and at different points within and behind the assembly. The images taken behind the layer, which would normally contain the microchannels, clearly show that the exiting beams diverge rapidly. The distance between the two beams increases from $0.45 \mathrm{~mm}$ at $1-\mathrm{mm}$ distance from the chip to about $2 \mathrm{~mm}$ at $5 \mathrm{~mm}$. The false-light intensity behind the collection microlens (where the detector would be placed) was mainly the result of an incompletely opaque aperture layer. Despite some excitation light passing between the two first microlenses, the intensity is still about 8 times weaker than on the sides (see Fig. 8). The excitation volume would be given here by the microchannel crosssection and the excitation spot dimension along the microchannel (i.e., the length of the cylindrical microlens). For a microchannel $110 \mu \mathrm{m}$ wide and $50 \mu \mathrm{m}$ deep and a spot length of $\approx 2.1 \mathrm{~mm}$, the excitation volume would be $\approx 8$ $\mathrm{nL}$.

The off-axis scheme was realized using the same kind of glass plates with cylindrical microlenses. Again the plates were aligned under a microscope and glued together with UV-curable glue. Figure 9 shows a sequence of photo-

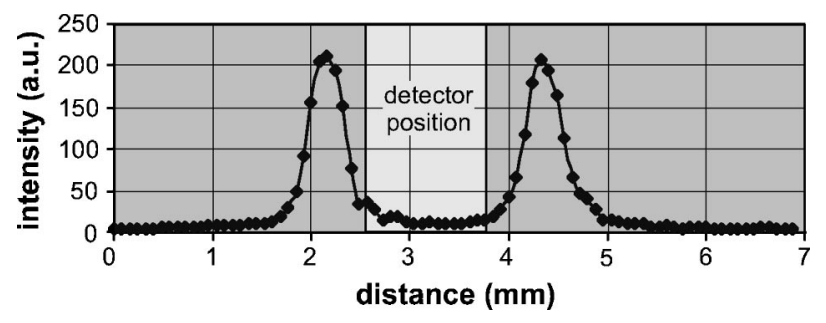

Fig. 8 Line plot of the intensity distribution in a plane situated $5 \mathrm{~mm}$ away from the focal plane. The scan was taken along the $y$ axis, normal to the optical axis.

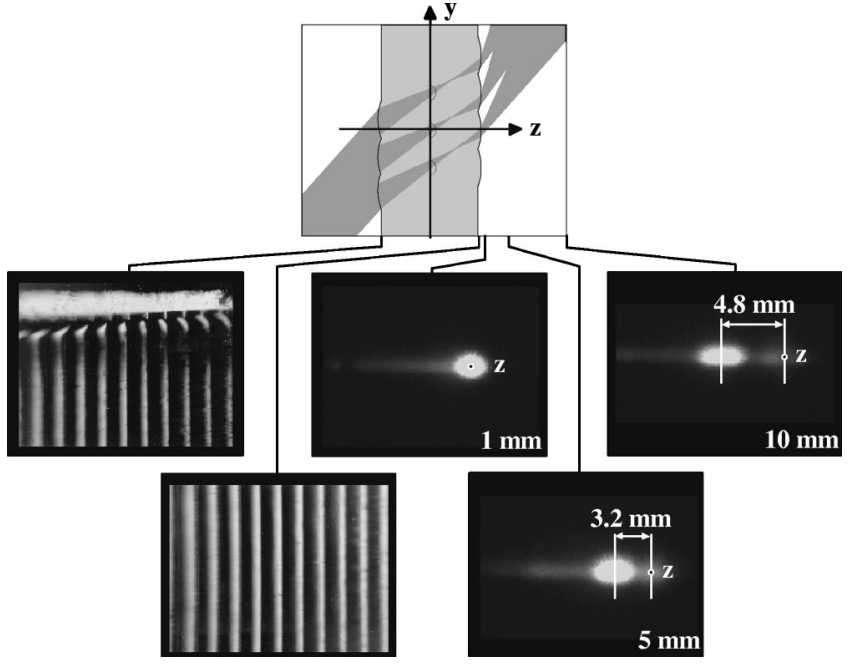

Fig. 9 Top picture: illumination scheme of the off-axis illumination system. Five bottom pictures show (from left to right): stacks of cylindrical microlens arrays, and exiting beams moving sideways from the detection axis at about 1,5 , and $10 \mathrm{~mm}$ away from the surface of the chip.

graphs of the microlens stack, the light distribution at the level of the second layer of microlenses, and the intensity distribution along the $z$ axis in perpendicular planes at various distances from the surface. The distance between the center of the exiting beam (light spot) and the $z$ axis (photodetector position) increased from 0 to $4.8 \mathrm{~mm}$ as the diffusing screen was moved from 1 to $10 \mathrm{~mm}$ away from the chip. As can be seen from Fig. 10, the intensity at the detector position is about 4 times weaker than in the exiting beam positions at a distance of $5 \mathrm{~mm}$ from the chip. The false light is mainly generated by the light passing in the gap between the microlenses or at the rim of the microlenses. The integration of apertures could increase the separation. As in the previous system, the excitation volume would be given by the microchannel cross section and the excitation spot dimension along the microchannel; the result is the same: $\approx 8 \mathrm{~nL}$.

The system with the central stop was realized on a single glass wafer (diameter $100 \mathrm{~mm}$ and thickness $0.5 \mathrm{~mm}$ ). The photograph on the left in Fig. 11 shows microlenses with stops at their centers. Then, in the next photographs, the intensity distribution of the excitation beam passing through the system can be observed. The light is first focused onto a spot where the detection cell would be placed and then diverges as it leaves the chip. A dark circular area

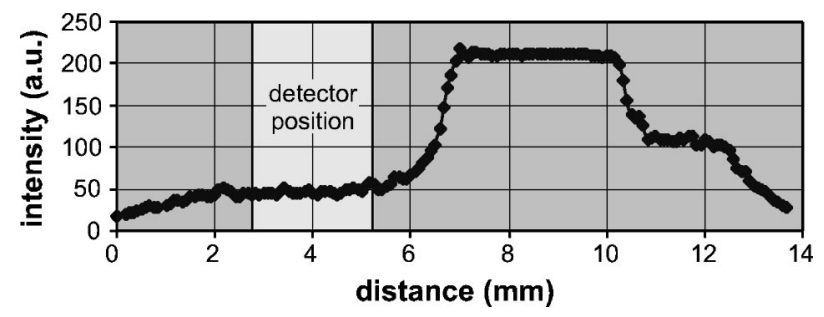

Fig. 10 Line plot of the intensity distribution in a plane situated 5 $\mathrm{mm}$ away from the focal plane. The scan was taken along the $y$ axis, normal to the optical axis. 


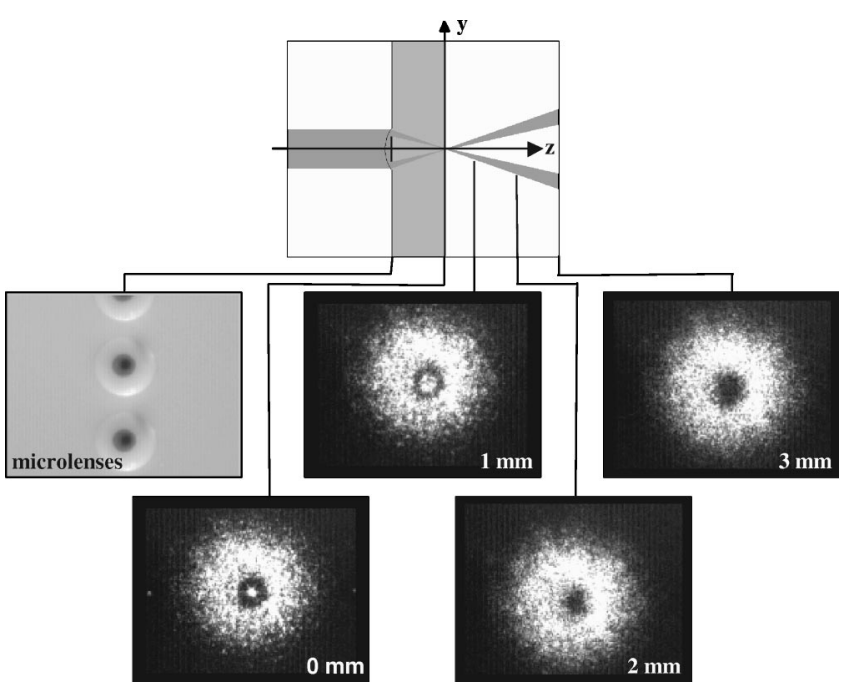

Fig. 11 Top picture: illumination scheme of the central-stop illumination system. Five bottom pictures show (from left to right): spherical microlens array with chromium stop, and exiting beams at 0,1 , 2 , and $3 \mathrm{~mm}$ away from the focal plane. The bright corona around the dark area is due to the light passing around the microlens.

with a radius of $0.4 \mathrm{~mm}$ is created in a plane perpendicular to the $z$ axis, $3 \mathrm{~mm}$ away from the surface of the chip. There, as shown in Fig. 12, the intensity at the detector position is between 6 and 7 times weaker than on the sides. As is clearly shown in Fig. 11, the light that is not focused by the microlens (bright outer ring) adds to the false light. Again the solution to this problem is the integration of apertures. Unlike the other systems, the excitation microlens is circular. The excitation volume is thus given by the microchannel depth and the excitation-spot radius (as long as the spot diameter does not exceed the microchannel width). For a spot radius of $\approx 40 \mu \mathrm{m}$ and a 110 - $\mu \mathrm{m}$-wide and 50 $\mu \mathrm{m}$-deep microchannel, the excitation volume would be $\approx 250 \mathrm{pL}$.

In terms of assembly, the beam-splitting excitation system is the most difficult, requiring two microlens layers. Therefore it is more complex and difficult to align than the other systems. On the other hand, nearly all the excitation light passing through the microlenses is usable to excite fluorochromes, which is also true for the off-axis system, but not for the central-stop system. In this latter case, if the stop is about three-fifths of the microlens diameter, only about $55 \%$ of the excitation light passing through the microlens is used. With regard to optical quality, the beam focused by the off-axis system has more aberrations. Thus,

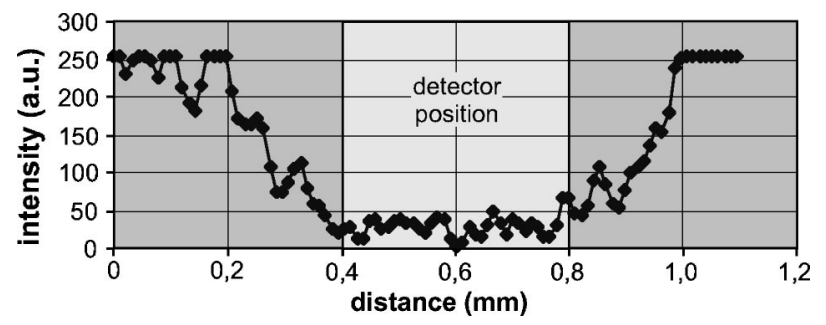

Fig. 12 Line plot of the intensity distribution in a plane situated 3 $\mathrm{mm}$ away from the focal plane. The scan was taken along the $y$ axis, normal to the optical axis.

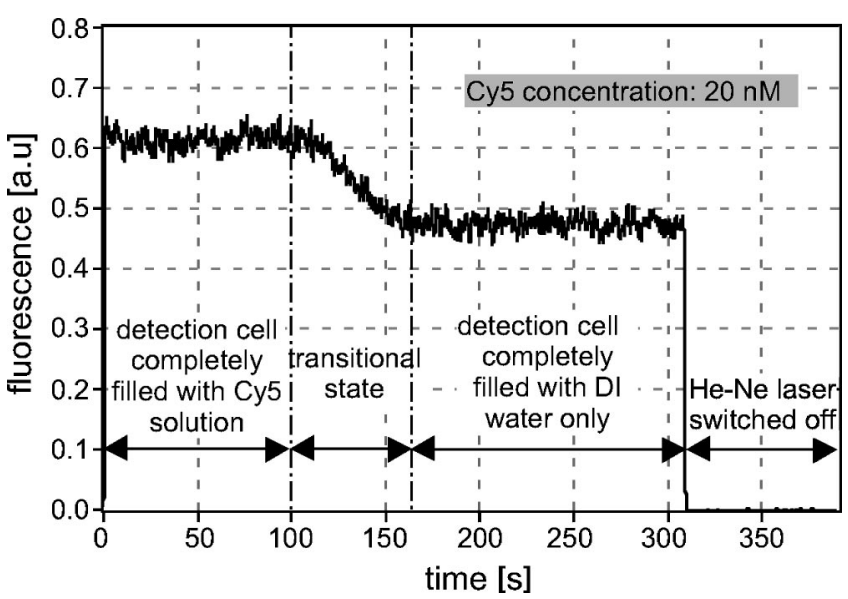

Fig. 13 Fluorescence intensity versus time. After $100 \mathrm{~s}$ the Cy5 dye concentration in the detection cell is reduced from $20 \mathrm{nM}$ to 0 (deionized water only). For comparison, the signal level without light (He-Ne laser switched off) is shown.

it may be more difficult to control false light within the chemical chip, which could decrease the sensitivity. One solution would be to use elliptical microlenses to partly compensate for the influence of the incident angle. This type of microlens can be realized with the fabrication technique described in Sec. $4 .^{22}$

Finally, the off-axis illumination system was chosen to be integrated onto a chemical chip composed of two Pyrex glass wafers, containing 50- $\mu \mathrm{m}$-deep and $110-\mu \mathrm{m}$-wide microchannels. The spherical photoresist microlenses were $310 \mu \mathrm{m}$ wide and about $33 \mu \mathrm{m}$ high. Two 400- $\mu \mathrm{m}$ pinholes in an aluminum foil, centered with respect to the refractive microlenses, were glued on both sides of the chip to block light not passing through the microlenses. Unfortunately, the diameter of the pinholes was slightly larger than the microlens diameters. Thus, they did not completely block the light passing around the rim of the microlenses, resulting in a reduction of the separation between the fluorescent light and the transmitted light. For this reason, the pinholes will be microfabricated for future systems. The excitation was provided by a $10-\mathrm{mW} \mathrm{He}-\mathrm{Ne}$ laser with an incident angle of $45 \mathrm{deg}$. Solutions of Cy5 at concentrations ranging from $50 \mu \mathrm{M}$ to $10 \mathrm{nM}$ in a solution of $50-\mathrm{mM}$ phosphate buffer ( $\mathrm{pH}$ 7.4) were used. The fluorescence light collected and collimated by the second spherical microlens (NA 0.23) was filtered with the help of an interference filter (Omega Optical XF46 EM) placed in front of a photomultiplier (Hamamatsu H5701-5). The detection and collection volume are both $\approx 250 \mathrm{pL}$. Figure 13 shows the signal detected for a concentration of $20 \mathrm{nM}$ (with a signal integration time of $1 \mathrm{~s}){ }^{26}$

\section{Conclusion}

Microfabrication techniques make possible the combination of microchemical chips with optical components of reduced size. The assembling of these elements in stacked form is conceivable. A complete microchemical analysis system with a large number of microchannels (which means that a large number of samples can be treated simultaneously) becomes possible due to the size and fabrication method of the microlenses-which is compatible with the microchip 
fabrication technology. Furthermore, we presented several solutions to realize an integrated detection system.

We have also successfully demonstrated the detection of a 20-nM solution of Cy5 fluorescent molecules in a microchannel by means of an off-axis illumination system.

This concept is highly adaptable and will permit the realization and the integration of micro-optical systems for $\mu \mathrm{TAS}$ in the near future.

\section{Acknowledgments}

The authors are thankful to Karl Fluir for his work on the microfluidic system and to Novartis Pharma AG. The project was funded by the Swiss priority program "Microand Nanosystems Technology", (MINAST).

\section{References}

1. D. J. Harrison, K. Fluri, K. Seiler, Z. H. Fan, C. Effenhauser, and A. Manz, "Micromachining a miniaturized capillary electrophoresisbased chemical analysis system on a chip," Science 261, 895-897 (1993).

2. E. Verpoorte, B. H. van der Schoot, S. Jeanneret, A. Manz, H. M. Widmer, and N. F. de Rooij, "Three-dimensional micro-flow manifolds for miniaturized chemical analysis systems," J. Micromech. Microeng. 4, 246-256 (1994).

3. A. T. Woolley, G. F. Sensabaugh, and R. A. Mathies, "High-speed DNA genotyping using microfabricated capillary array electrophoresis chips," Anal. Chem. 69, 2181-2186 (1997).

4. G. Ocvirk, T. Tang, and D. J. Harrison, "Optimization of confocal epifluorescence microscopy for microchip-based miniaturized total analysis systems," Analyst 123, 1429-1434 (1998).

5. A. Bruno, E. Baer, R. Völkel, and C. S. Effenhauser, "Micro-optical fluorescence detection for chip-based multiplexed analysis systems," in Micro Total Analysis Systems '98, D. J. Harrison and A. van den Berg, Eds., pp. 281-285, Kluwer Academic, Banff, Canada (1998), ISBN 0-7923-5322-6.

6. M. E. Warren, W. C. Sweatt, J. R. Wendt, C. G. Bailey, C. M Matzke, D. W. Arnold, S. A. Kemme, A. A. Allerman, T. R. Carter, R. E. Asbill, and S. Samora, "Integrated micro-optical fluorescence detection system for microfluidic electrochromatography," Proc. SPIE 3878, 185-187 (1999)

7. A. Manz, N. Graber, and H. M. Widmer, "Miniaturized total chemical analysis systems: a novel concept for chemical sensing," Sens. Actuators B 1, 244-248 (1990).

8. J. C. Fister III, S. C. Jacobson, L. M. Davis, and J. M. Ramsey, "Counting single chromophore molecules for ultrasensitive analysis and separations on microchip devices," Anal. Chem. 70, 431-437 (1998).

9. I. Kheterpal and R. A. Mathies, "Capillary array electrophoresis," Anal. Chem., 31A-36A (1999).

10. Z. Fan and D. J. Harisson, "Rapid injection and separation of amino acids with capillary electrophoresis systems micromachined on a glass chip," Anal. Chem. 66, 177-184 (1994).

11. M.-A.-A. Grétillat, F. Paoletti, P. Thiébaud, S. Roth, M. KoudelkaHep, and N. F. de Rooij, "A new fabrication method of borosilicate glass capillary tubes with lateral inlets and outlets," Sens. Actuators A 60, 219-222 (1996)

12. N. H. Chiem and D. J. Harrison, "Microchip-based capillary electrophoresis for immunoassays: analysis of monoclonal antibodies and theophylline," Anal. Chem. 69(3), 373-378 (1997).

13. J.-C. Roulet, R. Völkel, H. P. Herzig, E. Verpoorte, N. F. de Rooij, and R. Dändliker, "Multilayer systems combining microfluidic and micro-optical elements for fluorescence detection," J. MEMS (accepted for publication).

14. F. H. Kasten, "Introduction to fluorescent probes: properties, history and applications," in Fluorescent and Luminescent Probes for Biological Activity, W. T. Mason, Ed., pp.12-33, Academic Press, London (1993).

15. D. L. Taylor and E. D. Salmon, "Basic fluorescence microscopy," Methods Cell Biol. 29, 207-237 (1989).

16. D. C. Harris, "Fundamentals of spectrophotometry," in Quantitative Chemical Analysis, 5th ed., pp. 511-534, Freeman, New York (1999).

17. D. A. Skoog, F. J. Holler, and T. A. Nieman, "An introduction to ultraviolet/visible molecular absorption spectrometry," in Principles of Instrumental Analysis, 5th ed., pp. 300-325, Harcourt Brace, Philadelphia (1998).
18. J. R. Alcala, "Real-time chemical sensing employing luminescence techniques," in Topics in Fluorescence Spectroscopy, J. R. Lakowicz, Ed., vol. 4, pp. 255-292, Plenum, New York (1994).

19. D. J. S. Birch and R. E. Imhof, Topics in Fluorescence Spectroscopy, vol. 1, Plenum, New York (1991).

20. R. J. H. Clarck and R. E. Hester, Eds., Time-Resolved Spectroscopy, Advances in Spectroscopy, vol. 18, Wiley, Chichester (1989).

21. J. B. Pawley, Ed., Handbook of Biological Confocal Microscopy, 2nd ed., Plenum, New York (1995).

22. P. Nussbaum, R. Völkel, H. P. Herzig, M. Eisner, and S. Haselbeck, "Design, fabrication and testing of microlens arrays for sensors and microsystems,', Pure Appl. Opt. 6, 1-20 (1997).

23. A. Manz, D. J. Harrison, E. Verpoorte, and H. M. Widmer, "Planar chips technology for miniaturization of separation systems: a developing perspective in chemical monitoring," Adv. Chromatogr. 33, $1-66$ (1993).

24. H. P. Herzig, Ed., Micro-optics, Elements, Systems, and Applications, Taylor \& Francis, London (1997)

25. J.-C. Roulet, E. Verpoorte, R. Völkel, H. P. Herzig, N. F. de Rooij, and R. Dändliker, "Integration of micro-optical systems for fluorescence detection in mtas applications," in Micro Total Analysis Systems 2000, A. van den Berg, W. Olthuis, and P. Bergveld, Eds., pp. 163-166, Kluwer Academic, Enschede, The Netherlands (2000)

26. J.-C. Roulet, K. Fluri, E. Verpoorte, R. Völkel, H. P. Herzig, N. F. de Rooij, and R. Dändliker, "Micro-optic integration for fluorescence detection in $\mu$ TAS systems," in Transducers '99, vol. 1, pp. 120123, Sendai, Japan (1999)

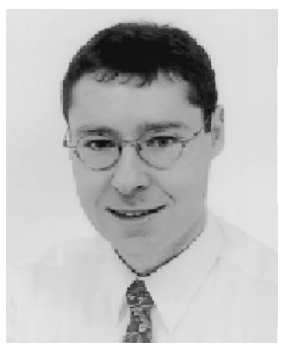

Jean-Christophe Roulet received his diploma in electrical and electronic engineering from the Swiss Technical College, Le Locle, in 1989 and his engineering degree in microtechnology from the Swiss Federal Institute of Technology, Lausanne, in 1995 From June 1995 to February 1996 he was with the development department of ASCOM Tech., Berne, Switzerland. He is currently a $\mathrm{PhD}$ candidate with the Institute of Microtechnology, University of Neuchâtel. His research interests include micro-optical systems for integrated chemical sensor applications ( $\mu$ TAS).

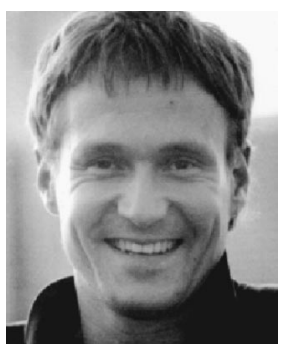

Reinhard Völkel received the diploma in physics in 1989 and the PhD in 1994, both from the University of Erlangen-Nuernberg, Germany, where he worked on diffractive optical elements for optical interconnects. $\mathrm{He}$ joined the Institute of Microtechnique at the University of Neuchâtel, Switzerland, in 1994, where he worked on micro-optics imaging systems, photolithography, and bio-sensors. Since 1999 he is in charge of the micro-optics department of Karl Süss KG located in Neuchâtel, Switzerland. He is involved in the development and fabrication of refractive microlens arrays for imaging and illumination purposes within photolithography systems.

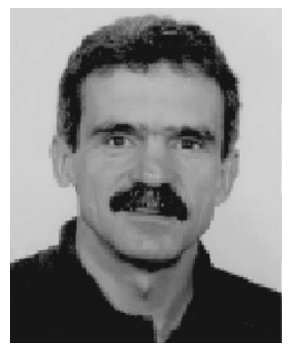

Hans Peter Herzig received his diploma in physics from the Swiss Federal Institute of Technology in Zürich, Switzerland, in 1978. From 1978 to 1982 he was a scientist with the Optics Development Department of Kern in Aarau, Switzerland, working in lens design and optical testing. In 1983, he became a graduate research assistant with the Applied Optics Group at the Institute of Microtechnology of the University of Neuchâtel, Switzerland, working in the field of holographic optical elements, especially scanning elements. In 1987, he received his PhD degree in optics. He currently heads the micro-optics research group and is an associate professor at the University of Neuchâtel. Dr. Herzig is member of OSA and EOS and a board member of the Swiss Society of Optics and Microscopy. 


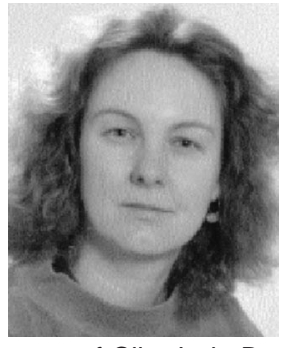

Elisabeth Verpoorte obtained her PhD in 1990 in analytical chemistry at the University of Alberta, Edmonton, Alberta, Canada. Her dissertation was concerned with the use of ac impedance analysis for studying the permeability and behavior of dissociable species in PVC-based ionselective membranes. She went on to obtain a postdoctoral position, and later a permanent position as research scientist, in the Corporate Analytical Research Department of Ciba Ltd., Basel, Switzerland, working on the development of miniaturized total analysis systems ( $\mu$ TAS). In July 1996, she assumed a position as team leader in the group of Prof. Nico F. de Rooij at the Institute of Microtechnology, University of Neuchâtel, Switzerland, where her research interests continue to concentrate in the area of $\mu$ TAS for bioanalytical applications.

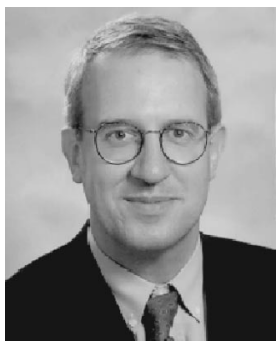

Nico F. de Rooij joined the Institute of Microtechnology of the University of Neuchâtel, Switzerland (IMT UNI-NE), as professor and head of the Sensors, Actuators and Microsystems Laboratory in 1982. From October 1990 till October 1996, he was director of the IMT UNI-NE. He lectured at the Swiss Federal Institute of Technology, Zurich (ETHZ), and since 1989 , he has been a part-time professor at the Swiss Federal Institute of Technology, Lausanne (EPFL). His research activities include microfabricated sensors, actuators, and microsystems. He was a member of the steering committee of the International Conference on Solid-State Sensors and Actuators and of Eurosensors. He acted as European Program Chairman of Transducers '87 and General Chairman of
Transducers ' $89 . \mathrm{He}$ is a member of the editorial boards for the journals Sensors and Actuators, Sensors and Materials, and the IEEE Journal of Microelectromechanical Systems.

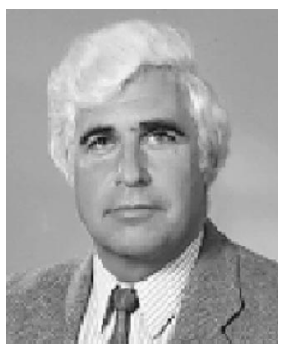

René Dändliker received his diploma in physics from the Swiss Federal Institute of Technology, Zürich, in 1963, his PhD in physics from the University of Berne, Switzerland, in 1968, and the Venia Legendi for applied physics from the Swiss Federal Institute of Technology, Zürich, in 1978. From 1963 to 1969 he was a graduate research assistant with the Institute of Applied Physics, University of Berne, working on gas and solid-state lasers. From 1969 to 1970 he was a research scientist with the Philips Research Laboratories, Eindhoven, Netherlands, working in the field of applied optics. From 1970 to 1978 he was a senior scientist and head of the Coherent Optics Group at the Brown Boveri Research Center Baden, Switzerland, where he was concerned with optical metrology applied to mechanics, such as laser Doppler velocimetry and heterodyne holographic interferometry. Since 1978 he has been a professor of applied optics with the University of Neuchâtel, Switzerland, and since 1989 also a professor of applied optics with the Swiss Federal Institute of Technology, Lausanne. His current research activities are in optical metrology, optical fibers and sensors, holography and optical computing, diffractive optical elements, and micro-optics. Dr. Dändliker was president of the European Optical Society from 1994 to 1996, and he is a vice president of the International Commission for Optics, a fellow of the OSA and the Swiss Academy of Engineering Sciences, an honorary member of the Swiss Society of Optics and Microscopy, a member of the SPIE, the French Society of Optics, the German Society of Applied Optics, and the European Physical Society, and an affiliate of LEOS/IEEE. 